\title{
"My daughter is unwell": Fears of a mother with a major psychiatric illness
}

P K D H J L De Silva Rajaratne, D M A Dahanayake

\section{Abstract}

Schizophrenia is a major mental illness with children of affected individuals experiencing multiple challenges, which are often overlooked by professionals. These children are at risk of mental health issues due to multiple reasons including genetic predisposition, poor attachment in relationships and chaotic family environments. We describe an unusual presentation of a child whose mother was suffering from schizophrenia.

Key words: parental mental illness, schizophrenia, parent-child relationship, psychosis in children

SL J Psychiatry 2021; 12(2): 28-30

\section{Introduction}

Schizophrenia is a major mental illness causing a significant impact on sufferers and families (1). Children of parents with schizophrenia feel emotionally detached from affected parents, fear the uncertainty of illness and crave normalcy (1). Their needs are often overlooked by professionals caring for parents, leading to them be referred to as 'hidden children' (1). There is limited evidence related to the impact of schizophrenia on the family, especially children in South Asian countries such as Sri Lanka (2).

There is a significant heritable component to schizophrenia, with $80 \%$ with childhood onset schizophrenia exhibiting a positive family history (3). However, 'psychotic' symptoms in children of parents with schizophrenia do not necessarily portend impending schizophrenia (4). When a child having a parent with schizophrenia develops psychotic symptoms, it is necessary to exclude organic causes, substance induced psychosis, depression, shared delusions, Munchausen syndrome by proxy and untreated delusions/obsessions in the parent $(4,5)$. It may be difficult to distinguish true hallucinations and delusions from imaginative play and a child may have limited understanding and skills to express internal experiences (3). Children with underdeveloped language skills, may mimic disorganized speech and thought characteristic of schizophrenia (3). This case report describes a mother who is on treatment for schizophrenia who presented with concerns that her daughter is developing a psychotic illness. This presentation was unusual due to the underlying factors contributing to her concerns and illustrates the impact of lived experience of a mental illness on a mother's worries.

\section{Case Report}

A 12-year-old girl was brought by her mother to the child psychiatry clinic requesting urgent psychiatric evaluation. She reported that the child had been smiling to self and socially withdrawn for several weeks. On detailed evaluation, no features of a major psychiatric disorder were found. The child was bored due to being confined to home for several months due to the COVID-19 pandemic. However, she continued to function at a satisfactory level, completing school assignments and attending to hobbies.

She was the youngest of three siblings. Her father was a professional and mother a retired teacher. The mother and her family had a dominating influence in the children's lives. There was a strong family history of schizophrenia, the mother and maternal uncles being on treatment for the condition. The mother reported that her eldest daughter aged 16 years was on treatment for schizophrenia and that she developed symptoms during early teens. The mother was prescribed clozapine. Both the mother and sister (who was on fluoxetine) were compliant with treatment and in remission.

Despite reassurance that the girl does not have symptoms of a mental illness, the mother kept bringing the girl repeatedly to the clinic, reporting that she was having "attacks" and demanding further assessments 
and initiation of medication. The mother was extremely anxious that the 'same fate' as hers might befall her daughter, unless urgent treatment was provided. She was however, amenable to reassurance, but was extremely vigilant, and would bring the child back at the slightest difference in behaviour, such as for example, being fearful immediately after watching a frightening movie.

The child was admitted for observation, where it emerged that she was bullied at home by the mother and the sister who called her a 'psycho'. She had been given several doses of risperidone covertly and erratically by her mother. She was found to be experiencing severe psychological stress and grieved the loss of a normal family life. No features of a psychotic illness were observed during the ward stay.

She denied fearful memories of her mother during the latter's past relapses. She was cared for mostly by her maternal grandmother, whom she recalled as being strict and emotionally distant. She felt closer to her mother than her father, and understood her mother's illness. She carried out domestic responsibilities due to this. She was embarrassed by her mother's behaviour at times, and refrained from bringing friends home. Considering the recent events, she felt resentful towards her mother and sister and abandoned by her family.

The psychiatric service caring for the sister revealed that she has a diagnosis of depression, although mother had concerns about a psychotic illness. A meeting was held with participation of the multidisciplinary team and child protection authorities. A second opinion was sought, which confirmed lack of evidence for a psychotic illness in the child. Concerns for the safety of the child were discussed, and she was returned to her family under strict supervision, with plans for follow up. The possibility of the presentation being due to a prodrome of a psychotic illness was considered. She did not have any features to suggest a psychiatric illness during followup visits, which her father regularly attended. Regular follow up sessions were offered, and psychological support given to the family to manage their distress.

\section{Discussion}

This illustrates a unique presentation of a child who has a parent with schizophrenia, highlighting the myriad biopsychosocial factors leading to such children being brought to mental health services. This mother was preoccupied with worries that her daughter would end up with a chronic mental illness similar to hers, inadvertently doing more harm than good to the child. Her worries stemmed from the love for her child, which her daughter acknowledged. The mother was the dominant parent in the family and her own family's influence was pivotal in worsening the mother's sense of responsibility and anxiety relating to a possible psychotic illness in her daughter. The strong genetic predisposition and reference to the older daughter as having schizophrenia, worsened the mother's concerns. She found it difficult to accept the diagnosis of depression in her older daughter, claiming that a diagnosis of schizophrenia was made previously and despite psychoeducation during subsequent visits, continued to have uncertainty regarding the elder child's diagnosis. nEvaluation confirmed that the mother's concerns were not a result of delusional beliefs and that she did not have any motives or gains to suggest Munchausen by proxy.

Although there have been many cases of shared delusions in children with parents with schizophrenia, presentations where genuine concerns predominate, fueled by adverse experiences of a mother with schizophrenia are rarely described (5). Hence, this area warrants further study to provide better support for these parents and children.

\section{Conflicts of interest}

None declared.

\section{Acknowledgments}

None.

\section{Author contributions}

PKDHJLDSR was responsible for patient assessment, literature review, concept, design and writing the manuscript. DMAD was responsible for assessment supervision, clinical management, concept, revision and approval of final version to be published.

\section{Consent to participate}

Informed written consent was obtained from the parents.

P K D H J L De Silva Rajaratne, Lady Ridgeway Hospital for Children, Colombo, Sri Lanka

D M A Dahanayake, Faculty of Medicine, University of Colombo, Sri Lanka and Lady Ridgeway Hospital for Children, Colombo, Sri Lanka

Corresponding author: P K D H J L De Silva Rajaratne Email: hasalarajaratne@yahoo.com

http://orcid.org/0000-0002-6921-7107 


\section{References}

1. Maybery D, Ling L, Szakacs E, Reupert A. Children of a Parent with a Mental Illness: Perspectives on Need. Australian E-Journal for the Advancement of Mental Health 2005; 4(2): 78-88.

2. Somers V. Schizophrenia: The impact of parental illness on children. Br J Soc Work 2007; 37(8): 1319-34.

3. Bartlett J. Childhood-onset schizophrenia: what do we really know? Health Psychol Behav Med 2014; 2(1): 735-47.

4. Stevens JR, Prince JB, Prager LM, Stern TA. Psychotic disorders in children and adolescents: A primer on contemporary evaluation and management. Prim Care Companion CNS Disord 2014; 16(2).

5. Nachane HB. Shared psychotic disorder in children of parents with untreated schizophrenia: A case series. Med JDY Patil Vidyapeeth 2021; 14(3): 